\title{
Resonant neurons and bushcricket behaviour
}

\author{
Barbara Webb · Jan Wessnitzer · Sarah Bush · Johannes Schul • \\ Jonas Buchli $\cdot$ Auke Ijspeert
}

Received: 4 September 2006/Revised: 20 November 2006/ Accepted: 25 November 2006/Published online: 19 December 2006

(C) Springer-Verlag 2006

\begin{abstract}
The resonant properties of the intrinsic dynamics of single neurons could play a direct role in behaviour. One plausible role is in the recognition of temporal patterns, such as that seen in the auditory communication systems of Orthoptera. Recent behavioural data from bushcrickets suggests that this behaviour has interesting resonance properties, but the underlying mechanism is unknown. Here we show that a very simple and general model for neural resonance could directly account for the different behavioural responses of bushcrickets to different song patterns.
\end{abstract}

Keywords Orthoptera $\cdot$ Phonotaxis $\cdot$ Pattern recognition $\cdot$ Resonance $\cdot$ Neural model

\section{Introduction}

The auditory communication systems of Orthoptera have been an important model system for exploring

B. Webb - J. Wessnitzer

Institute for Perception, Action and Behaviour,

School of Informatics, University of Edinburgh,

Edinburgh, UK

S. Bush · J. Schul

Division of Biological Sciences,

University of Missouri, Columbia, MO, USA

J. Buchli · A. Ijspeert

Biologically Inspired Robotics Group (BIRG),

School of Computer and Communication Sciences,

EPFL, Lausanne, Switzerland

B. Webb $(\bowtie)$

JCMB King's Buildings, Mayfield Rd, Edinburgh, UK

e-mail: bwebb@inf.ed.ac.uk how neural circuits control behaviour. Male crickets and bushcrickets produce stereotypic and species specific songs. Female response depends on recognition of the temporal pattern of song pulses. However, the mechanism by which this recognition occurs is still a matter of debate (Hennig et al. 2004). Behavioural experiments on the bushcricket Tettigonia cantans (Bush and Schul 2006) compared three alternative explanations for the female's band-pass preference for the pulse rate in male song: a circuit involving separate high-pass and low-pass filters; autocorrelation; and resonance. A stronger response to songs at integer fractions of the preferred frequency, and to other nonnatural songs that reinforce that rhythm, suggested that resonance is the best explanation.

Resonance in single neurons can occur as a result of intrinsic dynamics of membrane currents, and there is much interest in the significance of this property of neural processing (Hutcheon and Yarom 2000). Izhikevich (2001) proposed a simple model for a "resonate-and-fire" neuron and showed this has many interesting properties, including the capability of acting as a bandpass filter. The activity of the neuron is described by a complex variable $z$ whose time evolution is determined by the following differential equation:

$\dot{z}=I+(b+2 \pi \mathrm{i} \omega) z$

where $I$ is the input current, $\omega$ is the resonant frequency, and $b$ the rate of attraction to rest. The neuron "fires" when the imaginary part (considered as membrane potential) exceeds a threshold $a_{\text {thresh }}$. This is simply a damped oscillator, i.e. an input pulse will cause the membrane potential to start oscillating at the resonant frequency, with a gradually decreasing amplitude. The 
neuron fires if the peak of the oscillation exceeds a threshold-typically this requires two or more input pulses to occur either simultaneously, or at a relative interval close to the resonant frequency, so that the second input occurs at the peak of the previously induced oscillation. In the original formulation by Izhikevich, firing is followed by a non-linear reset to a new value, but this was excluded in our model to simplify analysis, as it was not found to significantly affect the results.

\section{Materials and methods}

We implemented Izhikevich's resonant neuron model, as described above, using the parameters $b=-30$, $\omega=25$ (the preferred pulse frequency for Tettigonia cantans), and $a_{\text {thresh }}=0.12$, simulated at a time-step of $1 \mathrm{~ms}$. The input to the neuron, $I$, was modulated to represent the different song patterns tested on the bushcricket. In the interest of simplicity we neglected all details of input signal shape, transduction processes, synaptic dynamics, and noise and simply used square pulses, that is, the input was either $I=0$ (for gaps between sound pulses) or $I \in[8,9,10,11,12]$ representing variation in sound amplitude of the pulse. The response measured is simply the number of times the neuron fires (crosses the threshold) during $1 \mathrm{~s}$ of song pattern. The results presented below show this response averaged across the different input amplitudes, and scaled so that the size of the maximum response corresponds to the maximum response seen in the behavioural data.

\section{Results and discussion}

In Figs. 1 and 2 we show that this simple model reproduces all the "resonant" properties of the bush cricket behaviour described in Bush and Schul (2006). Pulses of a constant duration (18 ms) played at frequencies from 8 to $67 \mathrm{~Hz}$, produce the expected peak at $25 \mathrm{~Hz}$, a second peak of half the size at $12.5 \mathrm{~Hz}$, and a smaller increase at $8 \mathrm{~Hz}$. Shuffled songs that have an average rate of $25 \mathrm{~Hz}$, but are created by combining songs with pulse periods of 18 and $40 \mathrm{~Hz}$ are less attractive. When the gap between pairs of pulses, or the length of the second pulse in each pair is increased, the song that best maintains the $25 \mathrm{~Hz}$ rhythm is more effective than either faster or slower songs.

A useful way to characterise the song preference of the insect more fully is to test it with songs that vary

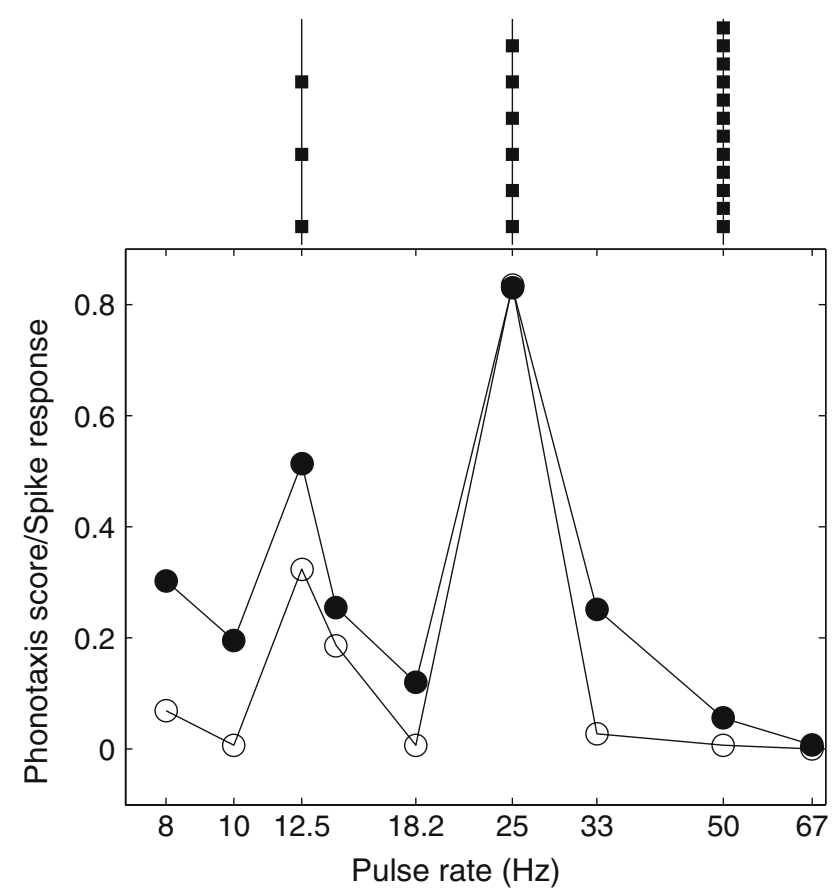

Fig. 1 Phonotaxis response of bushcricket (dark circles, replotted from Bush and Schul 2006) compared to firing rate of resonate-and-fire neural model (light circles) as an $18 \mathrm{~ms}$ pulse is played at different rates (for $67 \mathrm{~Hz}$ the pulse duration was $7 \mathrm{~ms}$ )

systematically in pulse and pause duration (Schul 1998). In Fig. 3 we show results for such tests on the model. This shows a good response over a large range of songs where pulse + pause $=40 \mathrm{~ms}$, a pattern of preference that has also been shown for $T$. cantans (Plewka 1993). It also predicts additional patterns for attractive stimuli, for example, that the animal should respond to songs where the pulse + pause $=80 \mathrm{~ms}-$ except that this response is diminished where pul$\mathrm{se} \approx$ pause. Note that in this plot the diagonal pulse = pause is equivalent to varying the frequency of pulses with a constant duty cycle of $50 \%$ instead of a constant pulse duration as in Fig. 1. Comparing the simulation result to the behaviour of the animal tested with a $50 \%$ duty cycle stimulus, as shown in Fig. 4 , we can see both that the overall shape of the tuning curve is very similar, and that the response to pulses at around half the frequency is considerably diminished in the insect, as the model predicts.

We can explain the effects of varying pulse and pause duration by considering the behaviour of the system as shown in the phase plane portraits in Fig. 5 . With a constant input, the behaviour is described by a logarithmic spiral, with a period of $2 \pi \omega$, winding towards a fixed point $z_{\mathrm{f}}$ located at $\operatorname{Re}\left(z_{\mathrm{f}}\right)=$ $I_{\frac{b}{b^{2}+\omega^{2}}}, \quad \operatorname{Im}\left(z_{\mathrm{f}}\right)=I_{\frac{\omega}{b^{2}+\omega^{2}}}($ marked as a square for $I=0$ and as an asterisk for $I=10$ in Fig. 5). For square wave 


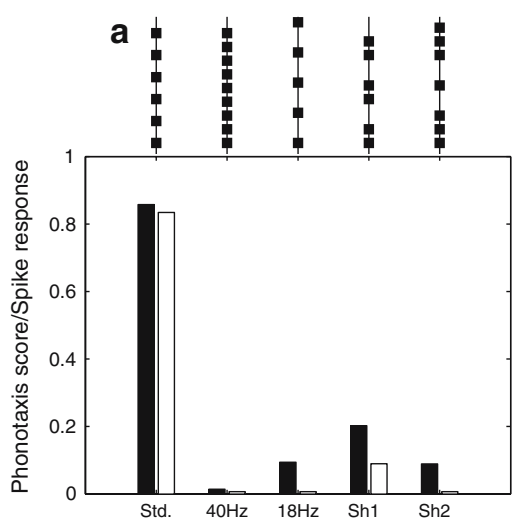

Fig. 2 Phonotaxis response of bushcricket (dark bars, replotted from Bush and Schul 2006) compared to firing rate of resonateand-fire neural model (light bars): comparing a standard song $(25 \mathrm{~Hz}), 40$ and $18 \mathrm{~Hz}$ with two songs (Sh-1 and Sh-2) created by shuffling the 40 and $18 \mathrm{~Hz}$ rates to give average rates of $25 \mathrm{~Hz}$;

input, the system switches between spiralling one fixed point and another. In order to pass above the threshold and produce spikes the system needs to maximise the radius of the spiral in the phase plane, which for a linear system corresponds to maximising its (phase) velocity $\dot{z}$. This means the switch between spirals needs to be timed so as to amplify or at least sustain $\dot{z}$. Geometrically, as the input is real, its effect is horizontal, and hence the switch will amplify $\dot{z}$ most if it occurs when $\operatorname{Re}(z) \approx \operatorname{Re}\left(z_{\mathrm{f}}\right)$ and $\operatorname{Im}(z)<\operatorname{Im}\left(z_{\mathrm{f}}\right)$ for the

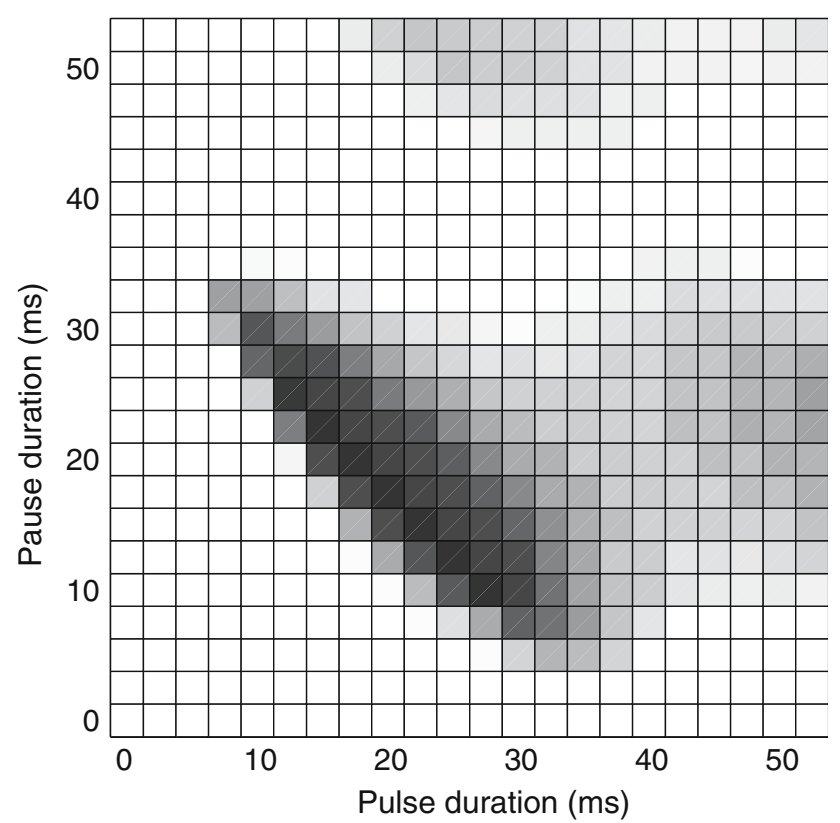

Fig. 3 Response of the model to varying the pulse and pause length. Darker squares indicate a stronger response

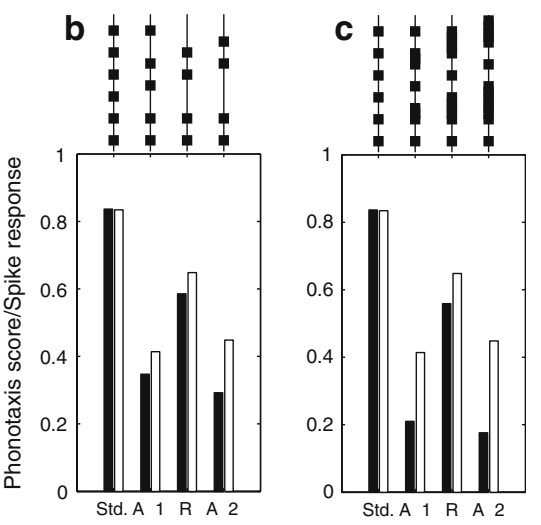

comparing standard song and rhythmic (R) stimuli created by $\mathbf{b}$ inserting gaps of $40 \mathrm{~ms}$ between song cycles or cextending every second syllable by $40 \mathrm{~ms}$, compared to arrhythmic (A) stimuli created by inserting gaps or extending syllables by $20 \mathrm{~ms}$ (A-1) or $60 \mathrm{~ms}(\mathrm{~A}-2)$

positive change, and $\operatorname{Re}(z) \approx \operatorname{Re}\left(z_{\mathrm{f}}\right)$ and $\operatorname{Im}(z)>\operatorname{Im}\left(z_{\mathrm{f}}\right)$ for the negative change. In other words it should act in the appropriate direction at the top or bottom of the spiral, as shown by the arrows in Fig. 5a. Thus certain timing constraints of the input have to hold: (1) the dynamical system needs to evolve for (approximately) an integer number of complete cycles between onsets (2) the dynamical system needs to make (approximately) half a cycle (or half a cycle after an integer number of cycles) during each constant part of the

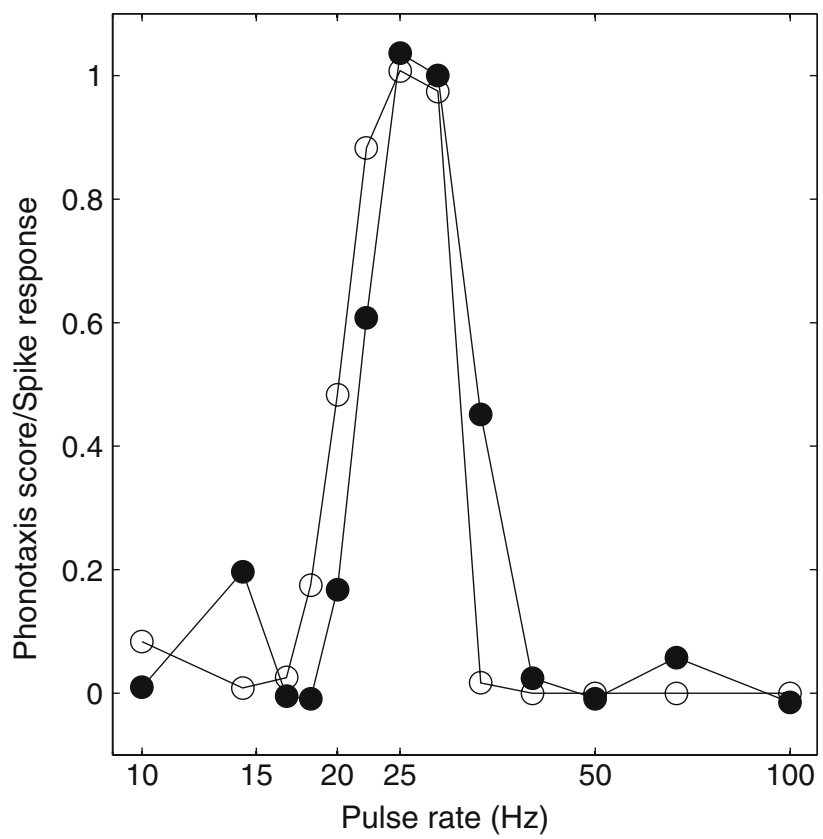

Fig. 4 Response of T. cantans (dark circles, data from Fritsch 2001) and the model (open circles) to varying the pulse rate with a constant duty cycle 


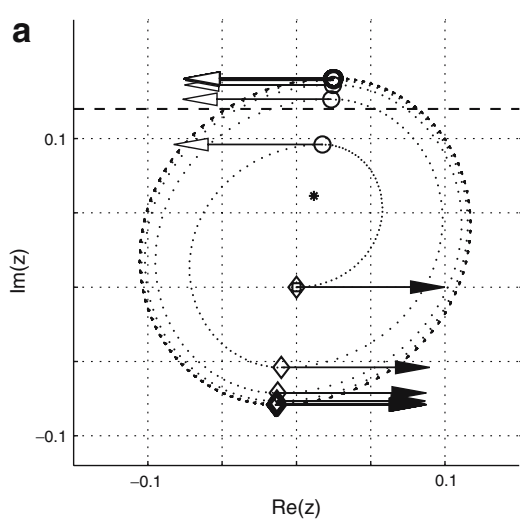

Fig. 5 Phase plane portraits of the system dynamics. The square marks the zero point for $I=0$ and the asterisk the zero point for $I=10$. The system switches between anti-clockwise logarithmic spirals about these two points, and spikes occur when it crosses the threshold (dashed line) $\operatorname{Im}(z)=0.12$. The radius of activity, and hence likelihood of spiking, increases if the changes in input (marked by diamonds for positive change, circles for negative

input. This translates into constraints on frequency and duty cycle, e.g. both conditions are satisfied by songs at around 25 or $12.5 \mathrm{~Hz}$ with a pulse duration around $20 \mathrm{~ms}$ (as seen in Fig. 5a) but the second condition is not satistified by songs at $12.5 \mathrm{~Hz}$ with a pulse duration around $40 \mathrm{~ms}$ (as seen in Fig. 5b).

The results presented here use the same model parameters for all experiments. The values for $b, a_{\mathrm{thresh}}$ and $I$ were determined by hand-tuning but varying these parameters does not produce significant qualitative changes in the results across different stimulus patterns. That is, a similar overall response pattern to that seen in Fig. 3 is observed, except that the overall number of spikes decreases with decreased $I$, increased $a_{\text {thresh }}$ or increased $b$, at the limits producing either saturated spiking or no response.

The results show that that neural resonance in a single neuron is a possible mechanism for temporal pattern recognition in $T$. cantans. It could also play a role in pattern recognition in other species of Orthoptera, or indeed in other animals. It shows that resonant properties of neurons could have a direct role in perception. At the same time, detailed modelling of membrane currents is not necessary to reproduce the effects: a basic model of damped resonance is sufficient in this case. It is possible that the resonance could be a property of a neural circuit rather than an individual neuron, although there is some evidence that an identified neuron in the auditory system of the cricket Teleogryllus oceanicus does show a resonant response (Hennig et al. 2004). It is interesting to note that the

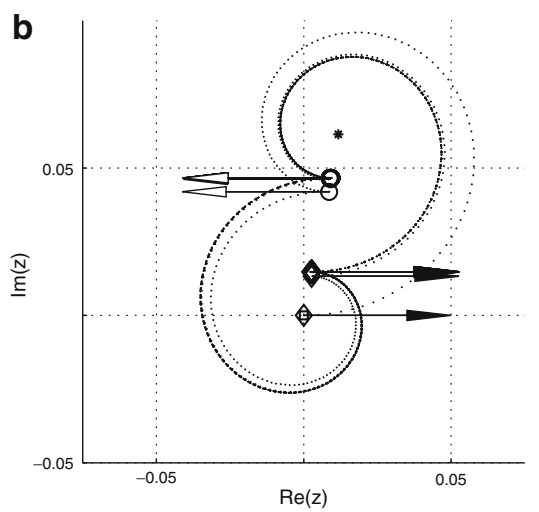

change) occur at times that increase or sustain the phase velocity $\dot{z}$, as seen on the left for a square wave at $25 \mathrm{~Hz}$ with a $50 \%$ duty cycle. Otherwise they tend to act against the phase velocity, reducing the spiral radius, as seen on the right for a square wave at $12.5 \mathrm{~Hz}$ with a $50 \%$ duty cycle (note different scale). This accounts for the dependence of the results on both frequency and duty-cycle of the input pattern

same model system could be driven by a constant tonic input to produce spikes at the pulse rate of the song, and thus could also serve as a pace-maker for the production of the song pattern by the male cricket.

Acknowledgments This work was carried out while B.Webb was a visiting professor at EPFL, Lausanne. Jan Wessnitzer is supported by the EC Sixth Framework SPARK project. Jonas Buchli and Auke Ijspeert are supported by the Swiss National Science Foundation.

\section{References}

Bush SL, Schul J (2006) Pulse-rate recognition in an insect: evidence of a role for oscillatory neurons. J Comp Physiol A 192:113-121

Fritsch M (2001) Selektive phonotaxis bei Tettigonia cantans: charaketerisierung der zeitlichen mustererkennung. Diplomarbeit. Friedrich-Alexander-Universität Erlangen-Nürnberg

Hennig RM, Franz A, Stumpner A (2004) Processing of auditory information in insects. Micros Res Tech 63:351-374

Hutcheon B, Yarom Y (2000) Resonance, oscillation and the intrinsic frequency preferences of neurons. Trends Neurosci 23:216-222

Izhikevich EM (2001) Resonate-and-Fire Neurons. Neural Networks 14:883-894

Plewka R (1993) Zur erkennung zeitlicher gesangsstrukturen bei laubheuschrecken: eine vergleichende untersuchung der arten Tettigonia cantans und Leptophyes laticauda. Thesis, University of Frankfurt

Schul J (1998) Song recognition by temporal cues in a group of closely related bushcricket species (genus Tettigonia). J Comp Physiol A 183:401-410 